\title{
The Swine Flu pandemic in Nepal
}

\section{Neopane A}

Associate Professor, Department of Medicine, Kathmandu Medical College, Sinamangal

$\mathrm{T}$ he H1N1 influenza pandemic, popularly known as "Swine Flu" has gripped everyone with fear. Every time one hears, reads or views news about its mortality, shivers goes down ones spine. People are really worried. Our government has done something to relieve this anxiety by broadcasting awareness programmes in the media and conducting few incompletely participated meetings to disseminate awareness among the health professionals and to inform about the national guideline. But has it come out with a well defined protocol for patients coming to the health care providers? Has it prepared a clear cut step wise guideline, is a big question mark! As efficient clinicians it is our duty to not only diagnose the disease correctly but also to dispel fears and threat associated with it.

This pandemic is unique in the sense that it is caused by a novel mutated influenza A virus and was first detected in April 2009, in Mexico. This virus includes one gene derived from human influenza virus, one from avian influenza virus and two from the pig influenza virus to form a new influenza virus designated as Influenza A (H1N1). As humans were exposed for the first time and it caused quite a number of mortality among young people (10-45years) there was a sense of panic. Nepal saw its first case in June 2009 among people returning from the US. But now more and more community transmission is being detected and the number of cases has increased to more than 63. The government has declared two H1N1 related deaths to date. A reliable source dealing with the H1N1 lab test, recently mentioned that majority of the strain circulating among flu cases this winter was found to be the H1N1 strain. Though this is not an official statement, what we can infer from this is that there need be no panic for swine flu.

More and more data are supporting the fact that this virus attacks people with preexisting medical conditions and very young children. This group must be protected with vaccine which is already been introduced but yet to come to Nepal. Similarly pregnant ladies are another special group to be protected. The main issue with this influenza is to find community where it has already spread, prevent spread to new community, isolate cases and spread awareness about the simple prevention methods as given in our national guidelines.
Going back to people who seek medical advice and present with acute febrile illness " how should a clinician proceed". Most of the cases are mild with symptoms like cough running nose, headache and can be sent home with advice on wearing mask, keeping in isolation, taking analgesics, and drinking a lot of fluid emphasising on hand washing and disposal of mask used by the patient. In others presenting with fever greater than $38{ }^{\circ} \mathrm{F}$ persisting for more than three days, patients with shortness of breath, and children with rapid breathing and altered consciousness we can proceed as follows:

Call it a suspected case and send nasopharyngeal swab for the available rapid test of Influenza A. Keep in isolation following all preventive measures

If the test is positive for Influenza A but is unsubtypable by reagents used to detect seasonal influenza, define the case as Probable. Send swab sample in a viral transport media for realtime RT-PCR, to the National public health lab, Teku. Start Tamiflu at the dose of $75 \mathrm{mg}$ twice a day for 5 days. Isolate the patient, nurse at 1 metre distance, wear mask and follow hand washing protocol.

If the test for PCR comes positive for H1N1 (Average 7 days) define the case as confirmed.

This confirmation is not for administration of Tamiflu but to find those exposed from this case and prevent exposure to other cases and to the community. Hopefully this step wise approach will mitigate the confusion among clinicians as how to proceed.

WHO considers the severity of this pandemic as moderate in view of the following:

- Overall level of severe illness is similar to seasonal flu that comes every year.

- Most of the affected have mild illness and recover spontaneously.

- Health care facilities are able to cope with the patient load.

Hence there need be no big panic for the "Swine Flu" at present. However recently there are news coming of viral mutation and resistance to the antiviral drugs which 
can make the pandemic worse. Hence viral monitoring for these possibilities and also for the possibility of new mutant virus needs to be ongoing.

\section{References}

1. Ministry of Health \& Family Welfare [homepage on the Internet]. India: Ministry of Health \& Family Welfare, Government of India [cited Nov, 2009]. Available from: www.mohfw.nic.in
2. World Health Organisation [homepage on the Internet]. Geneva: World Health Organisation [updated June 2009; cited Nov 2009]. Infection prevention and control in health care for confirmed or suspected cases of pandemic (H1N1) 2009 and influenza-like illnesses. Available from: http://www.who.int/csr/ resources/publications/infection_control/en/ index.html 